Research Article

\title{
Multisensor Information Fusion-Assisted Intelligent Art Design under Wireless Virtual Reality Environment
}

\author{
Dong Wenhao \\ Zhengzhou Business University, Zhengzhou City, Henan 451200, China \\ Correspondence should be addressed to Dong Wenhao; dongwenhao@hdu.edu.cn
}

Received 20 October 2021; Revised 24 November 2021; Accepted 4 December 2021; Published 31 December 2021

Academic Editor: Guolong Shi

Copyright (C) 2021 Dong Wenhao. This is an open access article distributed under the Creative Commons Attribution License, which permits unrestricted use, distribution, and reproduction in any medium, provided the original work is properly cited.

\begin{abstract}
Under the background of intelligent technologies, art designers need to use information technology to assist the design of art factors and fully realize the integration of art design and information technology. Multisensor information fusion technology can more intuitively and visually carry out a more comprehensive grasp of the objectives to be designed, maximize the positive effects of art design, and achieve its overall optimization and can also help art designers get rid of the traditional monolithic and obsolete design concepts. Based on multisensor information fusion technology under wireless virtual reality environment, principles of signal acquisition and preprocessing, feature extraction, and fusion calculation, to analyze the information processing process of multisensor information fusion, conduct the model construction and performance evaluation for intelligent art design, and propose an intelligent art design model based on multisensor information fusion technology, we discuss the realization of multisensor information fusion algorithm in intelligent art design and finally carry out a simulation experiment and its result analysis by taking the environment design of a parent-child restaurant as an example. The study results show that using multisensor information fusion in the environmental design of parent-child restaurant is better than using a single sensor for that; at the same time, using force sensors has a better environmental design effect than using vibration sensors. The multisensor information fusion technology can automatically analyze the observation information of several sources obtained in time sequence under certain criteria and comprehensively perform information processing for the completion of the decision-making and estimation tasks required for intelligent art design.
\end{abstract}

\section{Introduction}

The intelligent art design process is an effective way to improve the efficiency of art design, ensure the accuracy of art design, and improve the quality of art surface design. Sensors are indispensable for the intelligent art design [1]. Sensors used in the art design operation process can be divided into three categories: sensors used in the motion control process, such as position sensors, speed sensors, angular velocity sensors; sensors used in design process control, for example, force sensors and power sensors; and sensors used in the monitoring and diagnosis of the design process, such as vibration sensors and temperature sensors [2]. How to choose the appropriate sensor according to the different functions of the sensor in the process of art design and art design and use multisensor reasonably to improve the intelligent level of art design is the key issue; the current multisensor intelligent information fusion theory and technology are an effective way to solve this problem [3]. Since multisensor information fusion is realized by relying on the rule base, the key to intelligent art design lies in how to effectively establish the knowledge base, namely, the database and the rule base. The intelligent art design system based on multisensor information fusion technology collects sensor information, analyzes the data, and saves the data to the database. The experimental results show that the use of multisensor information fusion is better than the use of a single sensor for condition monitoring; it is pointed out that the use of force sensors is better than the use of vibration sensors [4].

Multisensor information fusion technology is an information processing process that uses computers to automatically analyze and synthesize the observation information of several sources obtained in time series under certain criteria to complete the required decision-making and estimation 
tasks [5]. Information fusion has three core aspects: information fusion is the process of processing multisource information at several levels, where each level represents different levels of information abstraction [6]. Multisensor information fusion technology is used to achieve interactive design, which can be integrated into parent-child restaurants to bring parents and children a full range of perception experiences. For example, under the trend of intelligent development, multisensor information fusion technology can more intuitively and visually carry out a more comprehensive grasp of the place to be designed and maximize the positive effects of art design, which can achieve art design. Multisensor data fusion actually integrates the data information from different sensors or sources in time and space to obtain a unified best estimate or description of the measured object or target; the use of multisensor data integration and fusion technology can combine data from multiple sources [7]. Sensor data and corresponding intelligence are analyzed and comprehensively processed, and the current model in the problem domain is established to provide preconditions for further reasoning and situation description [8].

Based on the summary and analysis of previous research works, this paper expounds the research status and significance of intelligent art design; elaborates the development background, current status, and future challenges of multisensor information fusion technology; introduces the methods and principles of signal acquisition and preprocessing, feature extraction, and fusion calculation; analyzes the information processing process of multisensor information fusion; conducts the model construction and performance evaluation for intelligent art design; proposes an intelligent art design model based on multisensor information fusion technology; performs the fusion of fuzzy set and evidence theory; analyzes the multisensor information fusion results of intelligent art design; discusses the realization of multisensor information fusion algorithm in intelligent art design; and finally carries out a simulation experiment and its result analysis by taking the environment design of a parent-child restaurant as an example. The study results of this paper provide a reference for further researches on the application of multisensor information fusion technology in intelligent art design. The detailed chapters are arranged as follows: Section 2 introduces the methods and principles of signal acquisition and preprocessing, feature extraction, and fusion calculation; Section 3 proposes an intelligent art design model based on multisensor information fusion technology; Section 4 discusses the realization of multisensor information fusion algorithm in intelligent art design; Section 5 carries out a simulation experiment and its result analysis; Section 6 is the conclusion.

\section{Methods and Principles}

2.1. Signal Acquisition and Preprocessing. Suppose the variances of $n$ sensors are $a_{1}, a_{2}, \cdots, a_{n}$, and the true value to be estimated is $A$; the measured values of each sensor are $A_{1}, A_{2}, \cdots, A_{n}$, which are independent of each other and are the element deviations of $X$ estimate; the weighting factors of each sensor are $b_{1}, b_{2}, \cdots, b_{n}$; then, the $A$ value and the weighting factor after fusion should meet:

$$
\sum_{i=1}^{n} A_{i}=\frac{\left(a_{1}, a_{2}, \cdots, a_{n}\right)^{T}}{\left(b_{1}, b_{2}, \cdots, b_{n}\right)^{T}} .
$$

The above is an estimation based on the measured value of each sensor at a certain moment. When the estimated value is constant, it can be estimated based on the average value of the historical data of each sensor.

As a function of defining parameters, the triangle membership function and the mapping determined are also not smooth for the center width parameter. The total confidence $B_{i}$ output of the rule base is defined as

$$
B_{i}=\sum_{i=1}^{n}\left(c_{i}-d_{i}\right) \log \frac{e_{i}}{f_{i}},
$$

where $c_{i}$ is the output of the $i$ th rule; $d_{i}$ is the output intensity of the $i$ th rule; $e_{i}$ is the sensor confidence of the $i$ th rule; and $f_{i}$ is the target decision value of the $i$ th rule.

Each node in the second layer represents a linguistic variable value; each node in the third layer represents a fuzzy rule, and its function is to match the antecedents of the fuzzy rule and calculate the practicality of each rule. The function of this layer is to calculate the membership function $C_{i}$ of each input component belonging to the fuzzy set of each linguistic variable value:

$$
C_{i}=\frac{\sum_{i=1}^{n}\left(g_{i}^{(j)}\right)^{1 / l}}{\sum_{i=1}^{n}\left(h_{i}^{(k)}\right)^{1 / l}},
$$

where $g_{i}$ is the variable parameter set; $h_{i}$ is the number of fuzzy divisions; $n$ is the dimension of the input; $j$ is the number of nodes; $k$ is the weighted sum of the rules; and $l$ is the normalized applicability.

According to the degree of abstraction of information and data, the information fusion of intelligent art design is divided into three levels: data layer, feature layer, and decision layer fusion. Intelligent art design only evaluates the fusion algorithm at the decision-making level; that is, after the identity recognition result output by each sensor is fused by the fusion module, the evaluation module evaluates the fusion result, so as to realize the evaluation of the fusion algorithm. When a fusion algorithm is developed, it is necessary to evaluate the art design; the simplest evaluation method is to find the recognition rate of the art design using multiple algorithms, and then, the evaluation module will compare the various art designs to the target [9]. The recognition results are compared with the preset standards, so that the performance of the art design can be known. The intelligent art design data generator module generates target data to simulate the art design data in the real environment; the sensor module adds noise to the target data sent from the data generator module to complete the target data preprocessing. The database module stores the original art design data generated by the data generator, the fused data, prior 
knowledge data, evaluation indicators, etc. Intelligent art design is the structure of dynamically mounting fusion algorithm, which provides an interface for the fusion algorithm. As long as the algorithm to be evaluated meets this interface standard, it can be directly mounted on the module for data fusion.

2.2. Feature Extraction and Fusion Calculation. The mode observed by the mode window can be imagined as a mode diagram, which describes the state of the process; the introduction of the mode window can facilitate the fusion of continuously changing signals, which is called time series fusion. In general, the expression of the sensor pattern vector $D_{i}$ can be written as

$$
D_{i}=\frac{p_{i}}{\sum_{i=1}^{n} o_{i}} \quad(i=1,2, \cdots, m)
$$

where $m$ is the number of sensors; $o_{i}$ is the number of data provided by each sensor in a mode window; and $p_{i}$ is the data provided by the $i$ th sensor to the mode.

Assuming that the fuzzy division number of each input component is determined in advance and the membership function adopts a bell-shaped function represented by a function, the parameters that need to be learned are mainly the connection weight $r_{i}$ of the conclusion network and the central value $s_{i}$ of the membership function of each node of the premise network width $t_{i}$; then, the error cost function $E_{i}$ is taken as

$$
E_{i}=\sum_{i=1}^{n} \frac{\left|r_{i}-s_{i}\right|}{\left|t_{i}-s_{i}\right|}-\sum_{i=1}^{n} \frac{\left|u_{i}-v_{i}\right|}{\left|w_{i}-v_{i}\right|},
$$

where $u_{i}$ and $w_{i}$, respectively, represent the expected output and actual output and $v_{i}$ is the membership function where the adjustable parameter is.

In order to facilitate the processing of the fusion degree between the observation data, the concept of membership function in fuzzy mathematics is prompted, and the observation value is mapped to the fusion degree function $F_{i}$ with the range of $[0,1]$; then, the sensor observed at time $x$ in the fusion degree of information data $F_{i}(x)$ and $F_{i}(y)$ is $G_{i}(x)$ :

$$
G_{i}(x)=\left[F_{i}(x), F_{i}(y)\right]=\left[\frac{F_{i}(x)}{F_{i}(x)+F_{i}(y)}, \frac{F_{i}(y)}{F_{i}(x)+F_{i}(y)}\right] .
$$

If $G_{i}(x)$ is closer to 1 , it is considered that the fusion of the two sensors is better, and the fusion of the observation data is higher; if the value of $G_{i}(x)$ is closer to 0 , the fusion of the two sensors is worse.

The distributed structure is the current mainstream information fusion structure. The reason is that it can obtain higher reliability and availability at a lower cost and can reduce the bandwidth of the data bus and the requirements for data processing; when a sensor is degraded, the impact on the performance and results of the entire multisensor data fusion is small [10]. It can gradually increase the number of automated functions to be realized and can adapt the system structure to the operating requirements of the control center. Therefore, the distributed structure has become the preferred solution for intelligent art design. The intelligent art design module integrates related design fusion algorithms for state fusion and attributes fusion of design objects and generates and outputs system design information. The simulation evaluation module compares the intelligent art design with the standard art design in the corresponding time period, calculates the root mean square error, and compares and evaluates the performance of the data fusion algorithm. The criterion of simulation evaluation is to have a small root mean square error of the target art design. The intelligent art design adopts an open structure, which can set various sensor information models and select various algorithms according to user requirements and can customize the algorithm library.

\section{Intelligent Art Design Model Based on Multisensor Information Fusion Technology}

\subsection{Information Processing Process of Multisensor} Information Fusion. The digitized electrical signal inevitably has some interference and noise signals due to the influence of random factors such as the environment. Through preprocessing, filtering and other methods are used to filter the interference and noise in the data acquisition process, and useful signals are obtained. The preprocessed useful signal is sent to the fusion center for data fusion; after feature extraction, data fusion calculation is performed on a certain feature quantity, and finally, the fusion result is output. The model first generates standard art design data from the standard art design module and stores it in the standard art design database, then adds different random noise data based on the standard art design data to form sensor simulation data and store it in the sensor database. The model reads the sensor data in the database and merges the node art design at the same time to form the node art design. This model transfers the art design to the fusion center, and the fusion center associates and synthesizes the various art designs and finally forms the system art design; it transmits the sensor data and the successfully fused art design data to the display control module in real time during the fusion process and show the integration process of art design. The simulation evaluation module reads the standard art design data and the fused art design data, evaluates the performance of each data fusion algorithm, and transmits it to the display control module to display the evaluation results [11]. Figure 1 shows the application framework of multisensor information fusion technology in intelligent art design.

The first step of multisensor information fusion is to determine the information source. The complex environment that may be involved in intelligent art design leads to too many uncertain factors in sensor information collection. Therefore, when selecting sensors, the models choose to use speed sensors, pressure sensors, and temperature. Sensors collect information from many aspects, and there are more sources of information, and multi-information fusion increases the accuracy of the identification results. In information fusion, the first level of information fusion is to fuse 


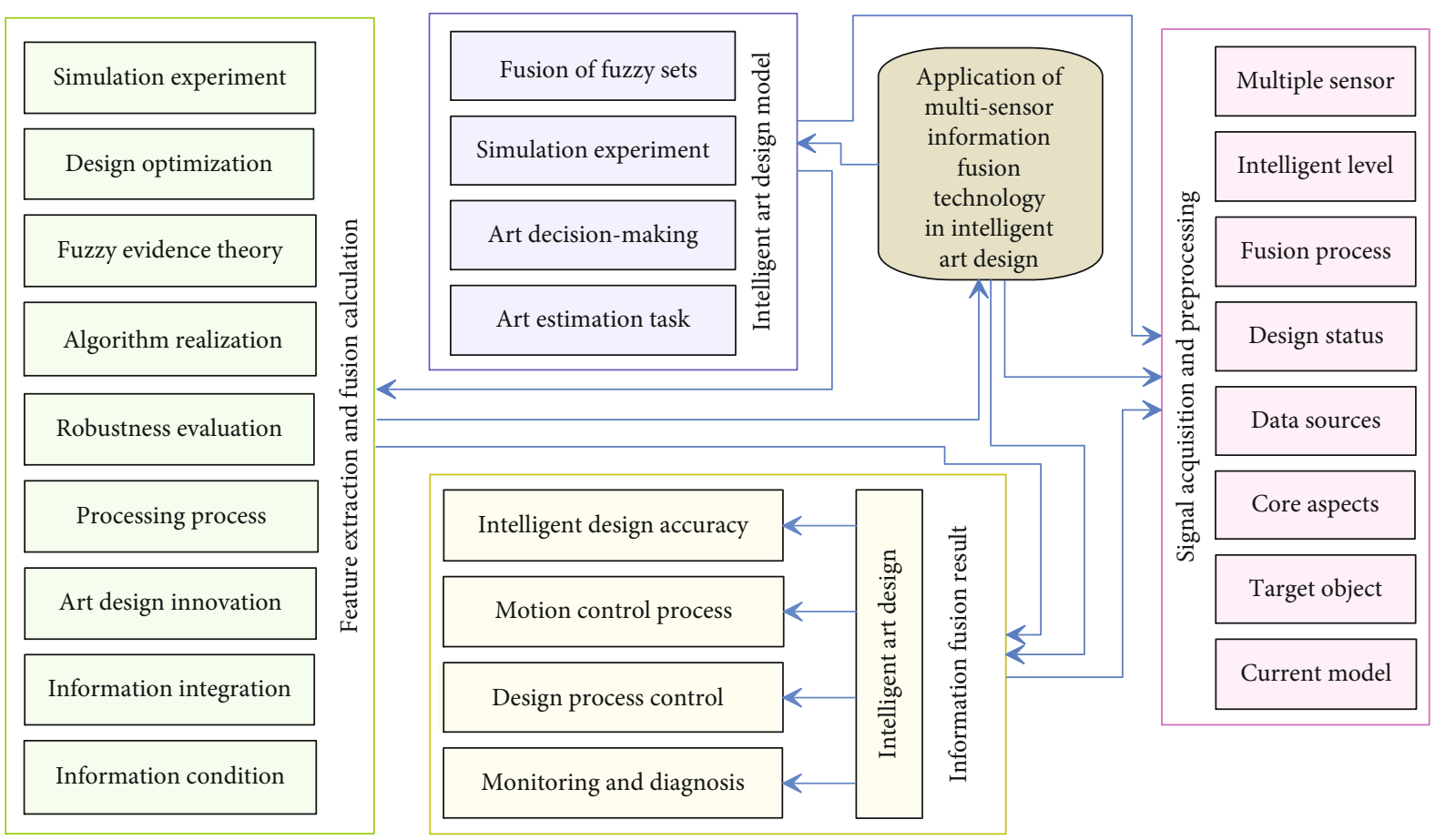

Figure 1: Application framework of multisensor information fusion-assisted intelligent art design under wireless virtual reality environment.

the information collected by multiple sensors of the same type, and the efficiency of art design and aesthetics is closely related, so the second level of information fusion is to collect different types of sensors. The received information is merged again to arrive at a final decision. In the process of completing information fusion, many modules have played a unique role. In the process of completing information fusion, the guidance of experts is extremely important, and guidance provided by experts constitutes a systematic expert knowledge base. The intelligent art design system based on multisensor information fusion technology collects sensor information, analyzes the data, and saves the data to the database. The mode adopted by the system is simple and easy to operate, and the safety can be greatly guaranteed.

The steps to achieve multisensor information fusion through fuzzy inference are as follows: first, convert the data information obtained by the multisensor into fuzzy sets and membership functions; then, build up the knowledge of experts in related fields into a fuzzy rule library; and then, use fuzzy operators to perform fuzzy inference and fusion of multisensor information and derive the final result. The fuzzy reasoning method can be used as an empirical rule to generate a fuzzy rule base based on the designer's choice when identifying rules and irregular design types [12]. After the intelligent art design converts the obtained distance and visual information into fuzzy sets and membership functions, based on the fuzzy rule library, an art type that meets the design experience can be derived. Compared with artificial intelligence algorithms such as neural network and ant colony algorithm, the design of controllers using fuzzy inference is relatively simple and more suitable for practical applications. In addition, based on fuzzy reasoning, intelli- gent art design can automatically identify regular or irregular art types and can improve the degree of intelligence of the art design system. Based on the above analysis, the intelligent art design scheme based on multisensor information fusion designed in this paper is distance sensor and visual information sensor as the environment perception framework, fuzzy inference as the information fusion method, and intelligent recognition of complex designs.

3.2. Model Construction and Performance Evaluation of Intelligent Art Design. Multisensor target recognition is actually a process of eliminating uncertainty, but this process also introduces information conflict. Due to the influence of various factors, the target recognition information provided by the sensor itself has uncertainty and ambiguity, and the quality of the information source also has a difference between good and bad. However, for multisensor target recognition systems that work in the field of intelligent art design, the environment is often complex and changeable, and it is not enough to rely on a priori information to determine the credibility of the sensor's local recognition information. The advantage is that the function is smooth for input variables and parameters. The disadvantage is that the function form is a transcendental function, which is expensive to implement. Infinite support complicates the inference process, so that the inference time is too long and the real-time performance of the system is limited (Figure 2). For different applications and application requirements, information fusion algorithms are different, including data-related technologies, estimation theories, and recognition technologies. Fusion calculation mainly verifies, analyzes, supplements, selects, and estimates state 


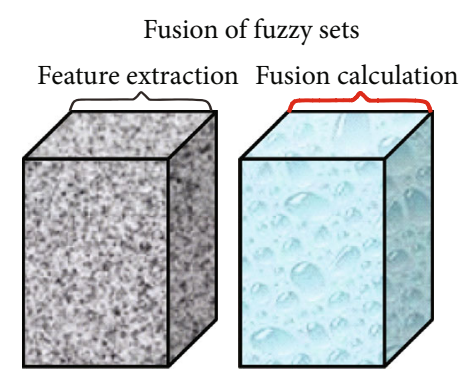

(a) Fusion of fuzzy sets

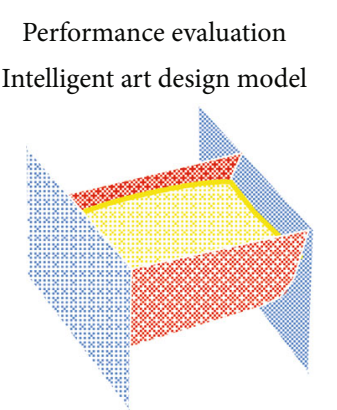

(b) Performance evaluation

FIgURe 2: Model construction and performance evaluation of intelligent art design based on multisensor information fusion technology under wireless virtual reality environment.

tracking of related observations from multiple sensors. It analyzes and synthesizes newly discovered irrelevant observations and generates a comprehensive situation and performs fusion calculations and modifies the overall situation based on multisensor observations in real time.

The intelligent art design based on multisensor information fusion technology can receive data from three different lower-level computers in a loop through the serial port. It can perform morphological planning according to the target position measured by the target sensor, adjust the design accuracy, and adjust the intelligent design speed according to the target distance measured by the ranging sensor. When the accuracy sensor finds that the current design object or process contradicts the design blueprint, the model will immediately stop operating and replan the design plan. Multisensor data fusion technology increases the dimension and confidence of the measurement, improves the detection performance, expands the coverage of space and time, and improves the reliability and maintainability of the system; this way, the system has better fault tolerance and is designed, when robustness is stronger, and the resource utilization rate in the system is also improved [13]. Feature extraction is required for the original information from multiple sensors, and the features can be various physical quantities of the measured object. In general, there are many parameters that need to be detected, such as temperature, pressure, and concentration. By using different acquisition channels of the data acquisition card, these data can be collected separately, and the detection data can be identified, and the detection values of various parameters can be assigned to different variables for storage.

The relationship between art design and intelligent platforms is similar to the relationship between humans and the brain. When humans make aesthetic judgments, aesthetic choices, and actions, they need the brain to perceive the surrounding environment and use destination information to form artistic plans. Therefore, an intelligent platform for art design actually needs a neural network to obtain, analyze, and integrate information, so as to form an artistic plan through the information instructions issued. By feeling the surrounding environment information in the process of fusion, it continuously forms new art design decisions, adjusts the current state, and achieves the goal blueprint. In the art design intelligent platform, it needs a systematic neural network, which is a neural network transmitted from human intelligence to artificial intelligence, so that artificial intelligence products can produce information judgment and decision-making effects like humans. In this process, we need to pay attention to a problem; that is, the information required by the intelligent platform of art design is diverse, and it also needs to form processing and decision-making information in a variety of information instructions, which requires the application of multisensor information fusion technology to art. In the design of the intelligent platform, with the help of multisensor information fusion, all the information of environmental characteristics is completely reflected in the neural network of art design [14].

\section{Realization of Multisensor Information Fusion Algorithm under Wireless Virtual Reality Environment}

4.1. Fusion of Fuzzy Sets and Evidence Theory. The main task of intelligent art design is to use the art index of the controlled system as the basis for designing and adjusting the parameters of the controller. Intelligent art design needs to consider the type of membership function and the overlap rate between different membership functions, the number of rules, and the precise calculation method (Figure 3). In the signal acquisition module, the middle value of different multisensor categories ranges from 0.3 to 0.6 , in which monitoring and diagnosis category is the highest with the value of 0.6. As for information fusion, the middle value of different multisensor categories ranges from 0.34 to 0.6 , in which motion control process is the highest with the value of 0.6. As for the signal preprocessing module, the middle value of that ranges from 0.37 to 0.6 , in which motion control process category is the lowest with the value of 0.35 . Since multisensor information fusion is realized by relying on the rule base, the key to intelligent art design lies in how to effectively establish the knowledge base, namely, the database and the rule base. Based on the art design experience, the model constructs training samples of the fuzzy neural network controller and applies the network parameter adjustment algorithm to perform offline training on the input membership function of the fuzzy neural network [15]. Once the error no 


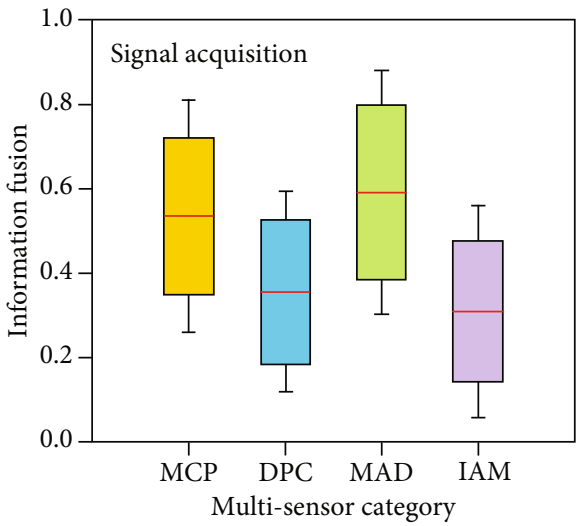

(a)

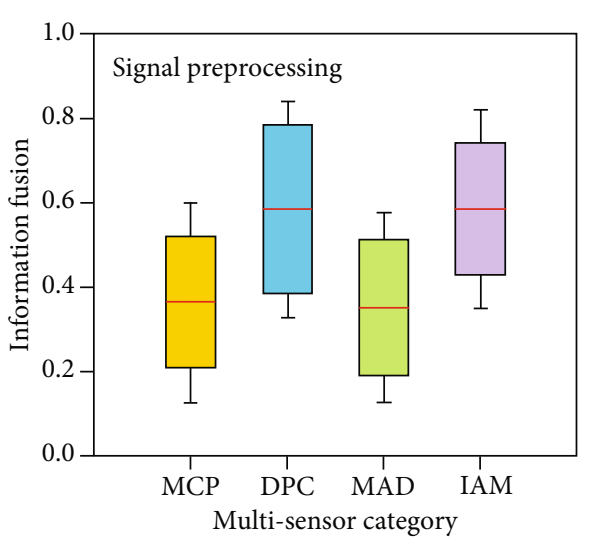

(b)

FIGURE 3: Information fusions of different multisensor categories in signal acquisition (a) and preprocessing (b). MCP: motion control process; DPC: design process control; MAD: monitoring and diagnosis; IAM: intelligent art modeling.

longer drops or even starts to rise, then it is considered that the current neural network has reached a state where it can stop training.

The work process of intelligent art design based on multisensor information fusion technology mainly includes the following: input is extracted and converted into fuzzy language as system input; based on expert knowledge, fuzzy antecedents are calculated and fuzzy subsequent are reasoned, and common rules are used to simulate humans. In the reasoning process, the fuzzy output is finally obtained. Through the above investigation and research, it is found that fuzzy neural network control technology is more adaptive than single fuzzy control and neural network and is suitable for intelligent art design. Usually, the environment of intelligent art design in the design process is dynamic and uncertain, so fuzzy control is used as the main control method. Also, because the environment of intelligent art design is dynamic, when the environment changes, the design object should have a certain learning ability and adapt to the new environment [16]. At this time, a neural network is needed to train the fuzzy control system parameters to achieve a better control effect. After the structure of the fuzzy neural network is determined, the number of fuzzy divisions of the input components is known in advance, but because the membership function and other structures are given based on expert experience, there will be differences in practical applications and low environmental adaptability, so the neural network trains, learns, and adjusts related parameters to achieve better control performance.

Multisensor data fusion is used to combine the redundant or complementary information of multiple sensors in time and space according to certain criteria and rationally control and use multiple sensors and their detected information, so as to obtain a consistent description of the measured object or explain. In order to ensure the accuracy and consistency of the information and remove the erroneous and useless information, it is necessary to process and synthesize the information from multiple perspectives, so as to obtain the internal relations and laws of various models, and finally realize the optimization of the information. According to the different levels of the fusion objects, information fusion can be divided into low-level, data-level or pixel-level, mid- dle-level, feature-level, high-level, and decision-level fusion. Data-level fusion can retain more original data information, but due to the incompleteness, uncertainty, and instability of the original data, it has higher requirements for the fusion error correction ability. Feature-level fusion extracts the required feature information from the raw data of each sensor, which facilitates data processing and analysis, but its accuracy is lower than that of data-level fusion. Decisionlevel fusion directly targets specific decision-making goals, makes full use of the various feature information of the target obtained by feature-level fusion, and gives concise and intuitive results, which has good real-time and fault tolerance, so it is the most widely used.

4.2. Information Fusion Results of Intelligent Art Design. In intelligent art design, art design is equivalent to a mapping of an input-output relationship. Input is the premise, and output is optimized as control input. Since neural networks can map arbitrary functional relationships, they can be used to realize art design. In addition, both the optimization and nonoptimization processes can be realized by neural networks, so that the entire fuzzy controller can be represented by neural networks. This kind of intelligent art design represented by neural network has many advantages, such as the amount of calculation has nothing to do with the amount of empirical knowledge, allows experience with a small amount of errors, and can perform parallel and distributed calculations. The key to intelligent art design is the acquisition of control rules [17]. Traditional methods are difficult to effectively acquire rules and adjust the membership function, and it is difficult to realize the learning function. The intelligent art design realized by neural network can allow neural network to learn existing experience rules and also can use network learning or clustering method to obtain control rules from input and output data and then use control performance indicators as guidance to control the control rules. By using different acquisition channels of the data acquisition card, these data can be collected separately, and the detection data can be identified, and the detection values of various parameters can be assigned to different variables for storage (Figure 4). 

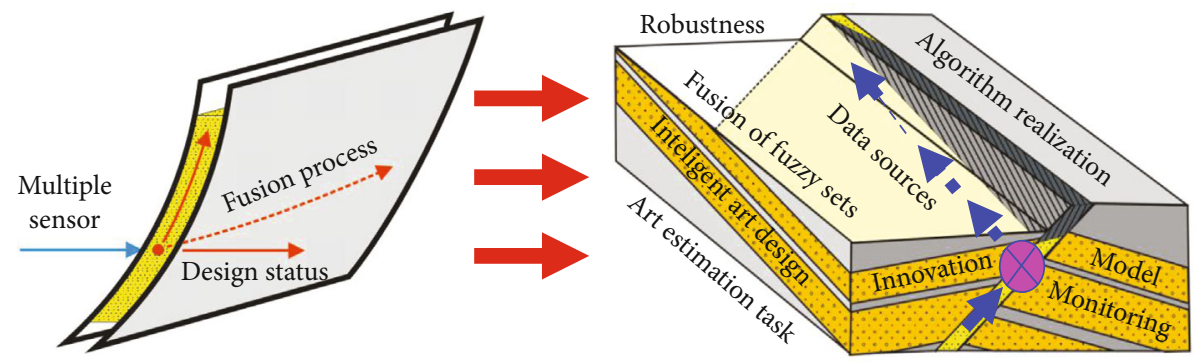

FIGURE 4: Information fusion results of intelligent art design under wireless virtual reality environment.

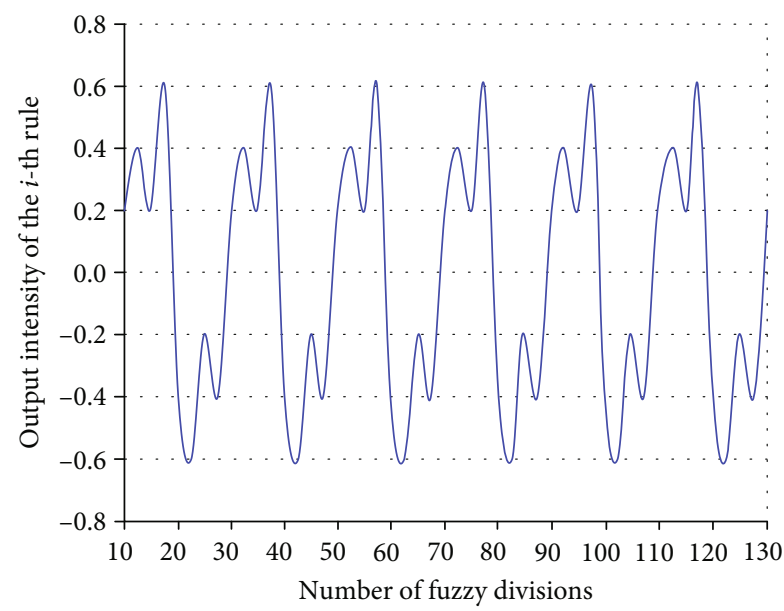

(a)

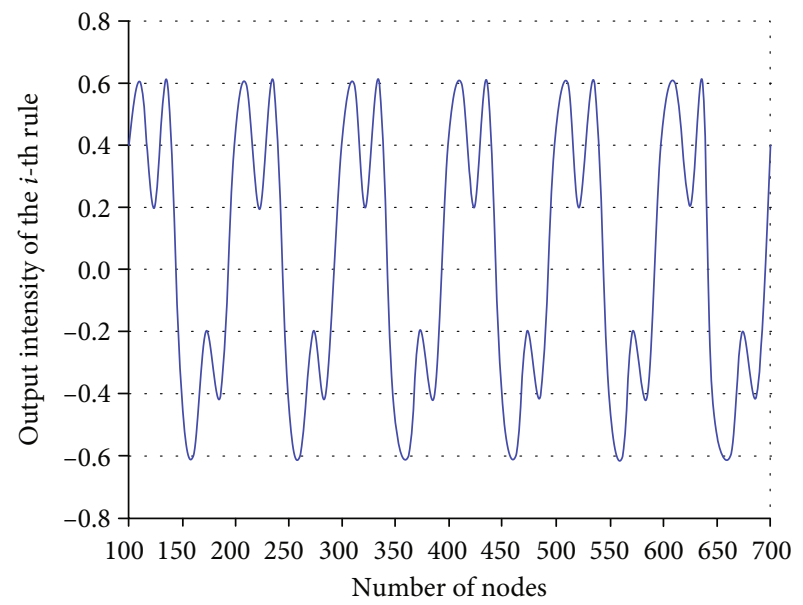

(b)

FIGURE 5: Relationship between output intensity and number of fuzzy divisions (a) and number of nodes (b).

Under the background of intelligent development, in order to realize the development and innovation of art design, it is necessary to require relevant art designers to have certain information technology awareness and be able to use information technology to assist environmental factors in the process of art design and fully realize the integration of art design and information technology. For example, under the trend of intelligent development, multisensor information fusion technology can more intuitively and visually carry out a more comprehensive grasp of the place to be designed and maximize the positive effects of art design, which can achieve art design [18] and overall optimization and can also enable related designers to introduce old and new and get rid of the traditional single-product and outdated design concept. In addition, in the context of intelligence, the use of digital technology for art design can also make art design more artistic (Figure 5). When using multiple sensors for information detection, because the measurement accuracy of each sensor is always different, if each sensor is treated the same, the detection data is processed and used without distinction, which will inevitably lead to inaccurate detection results. Therefore, it is necessary to selectively distinguish the importance of sensors according to the position of each sensor in the detection system and the accuracy of detection.

How to give life to intelligent art design has turned into two more basic problems. One is to make it reflect the corre- sponding, correct, scientific flow and transformation of the organism when under the action of external force. This kind of flow must be embodied in sensory attributes such as form, color, or hearing, so that it can be grasped by the living body; second, the dynamic shape as an organic whole must not only have the ability to interact with the external environment but also have the ability to reflect. At the same time, the manifestation of energy flow also implies the growth and disappearance of life. The linear method between one shape and another shape, due to the movement of each point in all directions, reflects a full change process in time, but the linear rate makes it slightly monotonous and dull. Correspondingly, in the experimental stage, these two elements are transformed into six dimensions that are easier to perceive and evaluate, which are fun, associative, continuity, interactivity, process, and energy flow. Among them, fun and optimization are the prerequisites for maintaining the continuous experience, continuity and process are the guarantee that the continuous experience will not be interrupted, and the interactivity and energy fluidity are the possibility for the continuous experience to be extended and continued.

\section{Simulation Experiment and Results}

5.1. Simulation Experiment Design. This paper takes the environmental design of a parent-child restaurant as an example to discuss the application of multisensor information fusion 


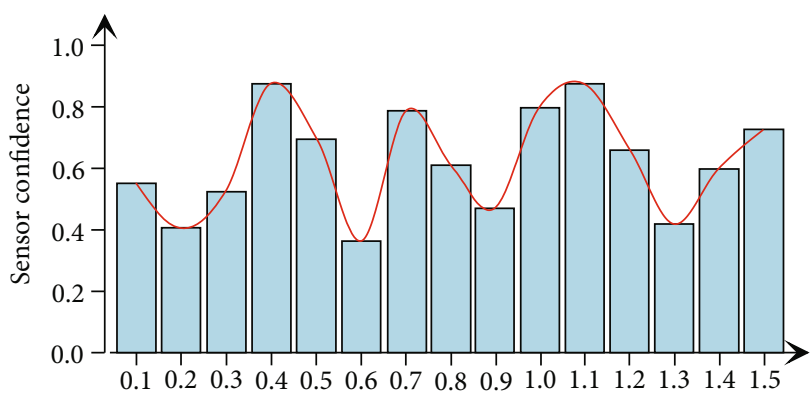

Weighting factor of each sensor

(a)

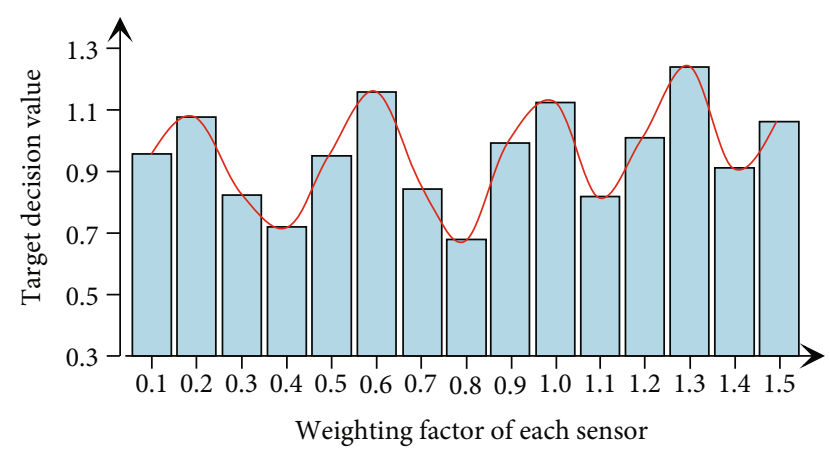

(b)

Figure 6: Sensor confidence (a) and target decision values (b) with different weighting factors of each sensor.

technology in intelligent art design. Parent-child restaurants began to design new service modes and new interactive forms of parent-child restaurants through the use of information technology and interactive design of smart devices to improve the comfort and richness of user experience. It uses multisensor information fusion technology to achieve interactive design, which can be integrated into parent-child restaurants to bring parents and children a full range of perception experiences. The development of multisensor information fusion technology has made interactive design present a rich and colorful appearance, and interactive design has been more widely used in indoor spaces. Especially the design of exhibition space has made a qualitative leap through the application of new digital media technology. User interaction is a key consideration in space design. The user's need for space is no longer a simple functional requirement but a higher standard of effective interaction. With the increase of the number of detections, the weighting factor is calculated according to the measurement data in each measurement, and its importance in the detection data processing is also reflected by the size of the weighting factor. In this way, based on the advantages of multiple sensors, the interference of environmental factors can be fully considered, and the influence of data with large deviations on the measurement accuracy can be reduced, and the accuracy of the measurement system can be improved.

The multisensor information fusion algorithm is used to combine the corresponding fusion algorithm to fully and completely reflect the information about the target object and environment obtained by multiple sensors. In the entire multisensor information system, the information we collect is diverse, and the information is sometimes more complicated. Therefore, this requires that the intelligent art design adopts the corresponding processing ability for the information fusion method, which not only has robustness but also has the ability to be parallel [19]. In addition, intelligent art design also has higher requirements for the coordination ability of different monitoring methods and technologies, the calculation speed and accuracy of the algorithm, the interface performance of the information recognition system and the preprocessing system, etc. The sample requirements of this information are for the entire system and have a very important role. Under normal circumstances, intelligent art design can also choose some other nonlinear mathematical methods as fusion methods, but these mathematical methods are required to have fault tolerance, associative memory, adaptive performance, and other capabilities. The highest level of information fusion is decision-level fusion, and the principle is that each sensor independently observes the measured target. After the target data is collected by the sensor, the sensor can independently correct the original data and eliminate redundant data.

5.2. Result Analysis. The state and observation fusion methods of intelligent art design include centralized fusion method and distributed fusion method, respectively. Among them, the centralized state fusion is the same as the centralized observation fusion algorithm; that is, the observation equations of all local sensors in the art design are merged into an augmented observation equation and then combined with the state equation to obtain a centralized global filter and finally obtain the global optimum untie. The distributed state fusion of art design is used to optimally weight the local filters corresponding to each sensor to obtain the distributed global filter and calculate it based on the local filter estimation error covariance matrix [20]. Multisensor data fusion will have a more sensitive response to the art design in the sampling set. If it is not eliminated, it will inevitably bring great errors to the data processing and cause the filter to fail. Intelligent art design is a very important part of the signal processing work process, which is of vital significance for improving the accuracy of data processing results and improving processing efficiency and quality (Figure 6). The main controller host of the monitoring center can communicate with other nodes on the bus and is mainly responsible for the monitoring and control of the entire detection system, such as system configuration, operating status display, concentration exceeding alarm, and coordination of the relationship between various parts.

If the data collected by a single sensor often contains noise and the sensor itself has a mean square error, there must be an estimation error in the estimated value obtained from the noisy data, and the error value is random. Using this type of data will make the system and the accuracy and real-time performance reduced. Therefore, multiple sensors of the same type can be used to measure the same data. Intelligent art design uses multiple sets of sensors to carry 


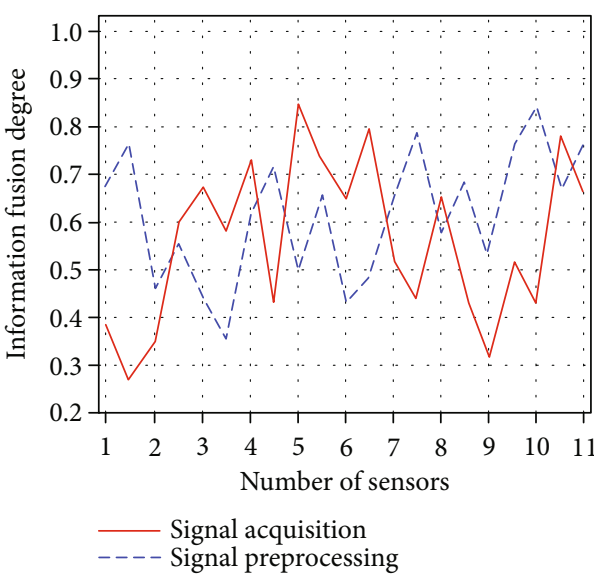

(a)

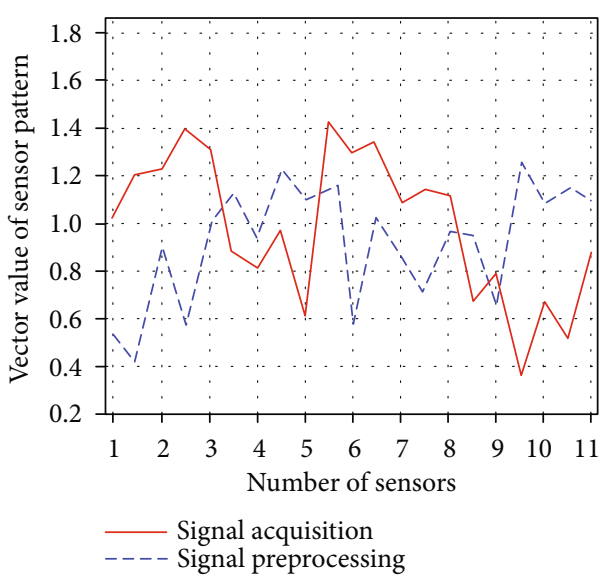

(b)

Figure 7: Information fusion degrees (a) and vector values of sensor pattern (b) at different numbers sensors.

out an adaptive weighted estimation data fusion algorithm. Information fusion performs multilevel processing of multisource information. Each level of processing abstracts the original data to a certain extent, which mainly includes the processing of information detection, calibration, correlation, and estimation. Information fusion can be divided into three levels according to the degree of abstraction of information processing in the fusion system: pixel level fusion, feature level fusion, and decision level fusion. According to the threshold value, the variance of each sensor is estimated, and the weighting coefficient of each sensor participating in the fusion is adjusted according to the principle of the minimum square sum of the weighting coefficient to ensure that the mean square error of the fusion data is kept to a minimum (Figure 7). This algorithm does not require sensor measure data information, and the algorithm estimation can theoretically prove the characteristic of linear unbiased minimum variance [21].

Data fusion technology has the advantages of improving the measurement accuracy of target parameters, eliminating the influence of interference, and overcoming its own time drift and aging. Multisensor information fusion technology is used to compress, format, standardize, batch, and sequence information from the art design information database to meet subsequent levels of estimation and the processor's requirements for calculation order and calculation volume [22]. For example, the original image collected by the camera is limited by random interference and various conditions, making the image obtained by the system imperfect. It is often necessary to use image processing technology to preprocess the obtained original image and perform noise filtering, distortion correction, and grayscale on the image. Degree correction is information preprocessing operations. Process evaluation is also called optimization fusion processing, which is used to modify the entire processing process in real time to achieve optimal utilization of resources, optimal sensor management, and optimal weapon control and improve the fusion effect of the system through feedback adaptive optimization information. This level mainly completes the monitoring and evaluation of the fusion process and guides how to obtain information, so as to achieve the best fusion effect. This level has contact with other levels, system outside, and operating personnel; its main functions include evaluation, fusion control, processing of special information source requirements, and task management.

\section{Conclusions}

This paper analyzes the information processing process of multisensor information fusion, conducts the model construction and performance evaluation for intelligent art design, proposes an intelligent art design model based on multisensor information fusion technology, performs the fusion of fuzzy set and evidence theory, analyzes the multisensor information fusion results of intelligent art design, discusses the realization of multisensor information fusion algorithm in intelligent art design, and finally carries out a simulation experiment and its result analysis by taking the environment design of a parent-child restaurant as an example. Multisensor information fusion technology is used to compress, format, standardize, batch, and sequence information from the art design information database to meet subsequent levels of estimation and the processor's requirements for calculation order and calculation volume. In intelligent art design, art design is equivalent to a mapping of an input-output relationship and input is the premise, and output is optimized as control input. The model first generates standard art design data from the standard art design module and stores it in the standard art design database, then adds different random noise data based on the standard art design data to form sensor simulation data and store it in the sensor database. The study results show that using multisensor information fusion in the environmental design of parent-child restaurant is better than using a single sensor for that; at the same time, using force sensors has a better environmental design effect than using vibration sensors. The multisensor information fusion technology can automatically analyze the observation information of several sources obtained in time sequence under certain criteria and comprehensively perform information processing for 
the completion of the decision-making and estimation tasks required for intelligent art design. The study results of this paper provide a reference for further researches on the application of multisensor information fusion technology in intelligent art design.

\section{Data Availability}

The data used to support the findings of this study are available from the corresponding author upon request.

\section{Conflicts of Interest}

The author declares that there is no conflict of interest.

\section{Funding}

This study received financial support through projects of prefectures, cities, departments, bureaus, and other government departments' research on green space landscape design of Zhengzhou from the perspective of National Central City Construction SKL-2019-1794.

\section{References}

[1] S. Serafin, C. Erkut, J. Kojs, N. C. Nilsson, and R. Nordahl, "Virtual reality musical instruments: state of the art, design principles, and future directions," Computer Music Journal, vol. 40, no. 3, pp. 22-40, 2016.

[2] S. A. al Hashimi, A. A. al Muwali, Y. Zaki, and N. Mahdi, "The effectiveness of social media and multimedia-based pedagogy in enhancing creativity among art, design, and digital media students," International Journal of Emerging Technologies in Learning, vol. 14, no. 21, pp. 176-190, 2019.

[3] T. S. Balint and P. Pangaro, "Design space for space design: dialogs through boundary objects at the intersections of art, design, science, and engineering," Acta Astronautica, vol. 134, pp. 41-53, 2017.

[4] R. K. Sawyer, "Teaching and learning how to create in schools of art and design," Journal of the Learning Sciences, vol. 27, no. 1, pp. 137-181, 2018.

[5] R. Gravina, P. Alinia, H. Ghasemzadeh, and G. Fortino, "Multi-sensor fusion in body sensor networks: state-of-theart and research challenges," Information Fusion, vol. 35, pp. 68-80, 2017.

[6] D. de la Iglesia, G. Villarrubia, J. de Paz, and J. Bajo, "Multisensor information fusion for optimizing electric bicycle routes using a swarm intelligence algorithm," Sensors, vol. 17, no. 11 , p. 2501, 2017

[7] W. R. Williams, "Attending to thevisualaspects of visual storytelling: using art and design concepts to interpret and compose narratives with images," Journal of Visual Literacy, vol. 38, no. 1-2, pp. 66-82, 2019.

[8] Y. Lu, H. Wang, F. Hu, B. Zhou, and H. Xi, "Effective recognition of human lower limb jump locomotion phases based on multi-sensor information fusion and machine learning," Medical and Biological Engineering and Computing, vol. 59, no. 4, pp. 883-899, 2021.

[9] K. Kim and M. Lee, "Flocking in interpretation with visual art design principles," Wireless Personal Communications, vol. 93, no. 1, pp. 211-222, 2017.
[10] Y. Wang, G. Zheng, and X. Wang, "Development and application of a goaf-safety monitoring system using multi- sensor information fusion," Tunnelling and Underground Space Technology, vol. 94, 2019.

[11] G. Sachdev, "Engaging with plants in an urban environment through street art and design," Plants, People, Planet, vol. 1, no. 3, pp. 271-289, 2019.

[12] M. Muzammal, R. Talat, A. H. Sodhro, and S. Pirbhulal, “A multi-sensor data fusion enabled ensemble approach for medical data from body sensor networks," Information Fusion, vol. 53, pp. 155-164, 2020.

[13] Y. M. Andreeva, V. C. Luong, D. S. Lutoshina et al., "Laser coloration of metals in visual art and design," Optical Materials Express, vol. 9, no. 3, pp. 1310-1319, 2019.

[14] J. Wang, D. Wang, S. Wang, W. Li, and K. Song, "Fault diagnosis of bearings based on multi-sensor information fusion and 2D convolutional neural network," IEEE Access, vol. 9, pp. 23717-23725, 2021.

[15] F. Li and J. Fei, "Gesture recognition algorithm based on image information fusion in virtual reality," Personal and Ubiquitous Computing, vol. 23, no. 3-4, pp. 487-497, 2019.

[16] A. Nazir, K. M. Abate, A. Kumar, and J. Y. Jeng, "A state-ofthe-art review on types, design, optimization, and additive manufacturing of cellular structures," The International Journal of Advanced Manufacturing Technology, vol. 104, no. 912, pp. 3489-3510, 2019.

[17] T. Li, H. Fan, J. García, and J. M. Corchado, "Second-order statistics analysis and comparison between arithmetic and geometric average fusion: application to multi-sensor target tracking," Information Fusion, vol. 51, pp. 233-243, 2019.

[18] Y. Zhou, L. Chang, and B. Qian, “A belief-rule-based model for information fusion with insufficient multi-sensor data and domain knowledge using evolutionary algorithms with operator recommendations," Soft Computing, vol. 23, no. 13, pp. 5129-5142, 2019.

[19] S. Yang and X. Wang, "Sparse representation and SRCNN based spatio-temporal information fusion method of multisensor remote sensing data," Journal of Network Intelligence, vol. 6, no. 1, pp. 40-53, 2021.

[20] Z. X. Liu, C. X. Xie, M. Xie, and J. Mao, "Mobile robot positioning method based on multi-sensor information fusion laser SLAM," Cluster Computing, vol. 22, no. S2, pp. 50555061, 2019.

[21] S. G. Hassan, M. Hasan, and D. Li, "Information fusion in aquaculture: a state-of the art review," Frontiers of Agricultural Science and Engineering, vol. 3, no. 3, pp. 206-221, 2016.

[22] B. Daass, D. Pomorski, and K. Haddadi, "Design of multisensor fusion architectures based on the covariance intersection algorithm-estimating calculation burdens," Journal of Intelligent and Robotic Systems, vol. 101, no. 4, pp. 1-16, 2021. 